Revue des patrimoines

28 | 2016

Le moulage. Pratiques historiques et regards contemporains

\title{
Le moulage. Pratiques historiques et regards contemporains
}

Éditorial

Christine Lancestremère, Jean-Marc Hofman et Yves Le Fur

\section{(2) OpenEdition}

Journals

Édition électronique

URL : http://journals.openedition.org/insitu/12790

DOI : 10.4000/insitu. 12790

ISSN : $1630-7305$

Éditeur

Ministère de la Culture

Référence électronique

Christine Lancestremère, Jean-Marc Hofman et Yves Le Fur, « Le moulage. Pratiques historiques et regards contemporains », In Situ [En ligne], 28 | 2016, mis en ligne le 15 mars 2016, consulté le 23 septembre 2020. URL : http://journals.openedition.org/insitu/12790 ; DOI : https://doi.org/10.4000/ insitu. 12790

Ce document a été généré automatiquement le 23 septembre 2020.

\section{(c) $(1)$}

In Situ Revues des patrimoines est mis à disposition selon les termes de la licence Creative Commons Attribution - Pas d'Utilisation Commerciale - Pas de Modification 4.0 International. 


\title{
Le moulage. Pratiques historiques et regards contemporains
}

\author{
Éditorial
}

Christine Lancestremère, Jean-Marc Hofman et Yves Le Fur

1 Souvent considéré comme éloigné de l'expression artistique, le moulage est longtemps resté une pratique d'atelier, un outil. Au XIX siècle, parallèlement à la diffusion massive de moulages de sculptures de tous styles, âges et civilisations, la demande scientifique généralise la production d'empreintes à titre documentaire et à des fins de diffusion. La circulation des moulages entre les musées européens est même officiellement encouragée lors de l'Exposition universelle de 1867, avec la ratification par 15 nations de la «Convention internationale pour la promotion universelle des reproductions d'œuvres d'art ", initiée par Henry Cole, premier directeur du South Kensington Museum. Aujourd'hui, au-delà de la réévaluation du document, déjà en œuvre pour la photographie, les moulages restent des témoignages d'artisans-mouleurs méconnus et d'œuvres parfois disparues. Cela vaut tout autant pour les collections de moulages ethnographiques, archéologiques, pédagogiques ou relevant du champ des beaux-arts. La reconnaissance de cette valeur documentaire tend peu à peu à affranchir les collections de moulages du statut d'ersatz auquel elles ont longtemps été confinées. 
Figure 1

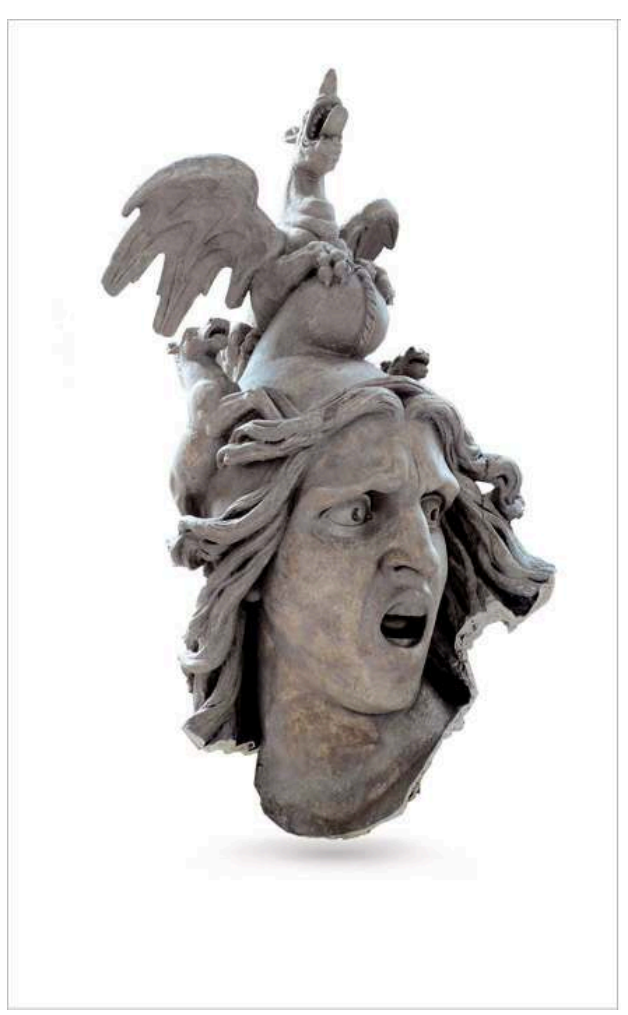

Moulage du Départ des volontaires (tête de la Renommée).

(c) CAPA/MMF.

2 La richesse des collections de moulages, en France comme dans les autres pays d'Europe, méritait l'approfondissement d'une réflexion dans ces domaines et une présentation des recherches les plus récentes. C'est dans cette perspective que la Cité de l'architecture et du patrimoine et le musée du Quai Branly organisaient les 14 et 15 novembre 2012 deux journées d'études interdisciplinaires sur le thème "Le moulage. Pratiques historiques \& regards contemporains». Une grande partie des contributions rassemblées dans ce numéro sont le fruit de ces rencontres auxquelles participèrent universitaires, responsables de collection de moulages, mouleurs, restaurateurs, artistes et jeunes chercheurs. La vitalité qui s'attache depuis plusieurs années à ce domaine d'étude et de recherche a suscité un enthousiasme dans la communauté scientifique qu'il aurait été dommage de brider : elle a pleinement justifié l'apport de nouvelles contributions à cette édition qui s'ordonne autour de quatre axes principaux.

Le premier axe, Le moulage, un art de la transmission, interroge le rôle de ces objets en tant que support de diffusion et d'enseignement de l'archéologie, de l'architecture, de la sculpture ou du dessin. Les collections de moulages universitaires en France et en Belgique y sont mises en valeur, de même qu'est explorée la relation entre la trace, l'empreinte et le vestige. 
Figure 2

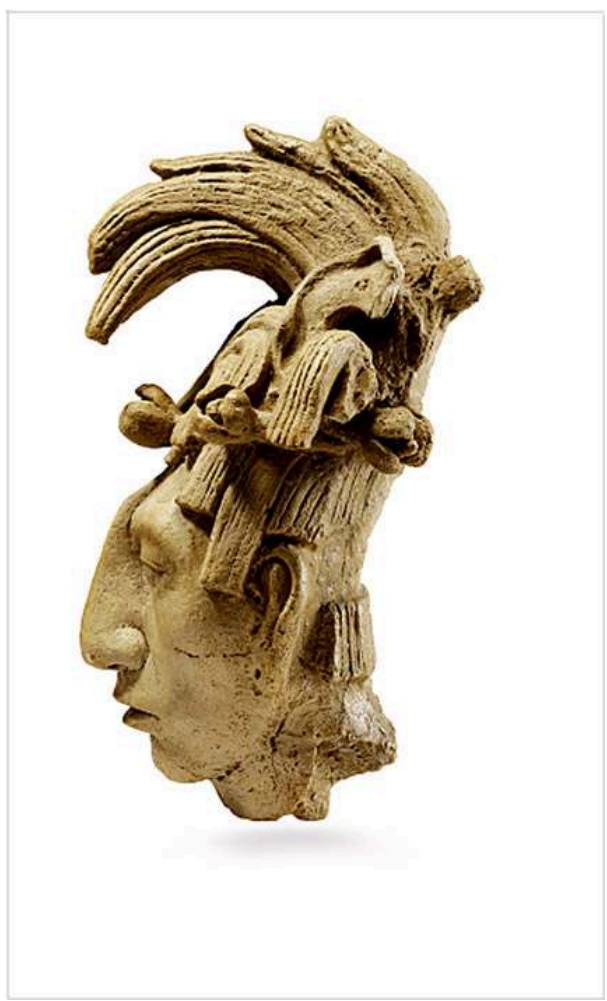

Moulage d'une sculpture du visage de K'inich Janaab' Pakal.

Phot. Germain, Claude. @ Musée du quai Branly.

4 Le second axe, Constitution des collections, études historiques, est une approche historique et matérielle des collections. Il aborde la création et la destinée de grandes collections de moulages, à l'instar de celles du musée des Arts décoratifs et industriels de Bruxelles, démantelée dans les années 1930. La réflexion sur les critères qui ont présidé à la constitution des collections de moulages est portée par l'exemple du musée de Sculpture comparée, dont le musée des Monuments français est l'héritier.

Les techniques de reproduction d'œuvres en volume, depuis la prise d'empreinte à la terre jusqu'à l'usage des techniques numériques les plus perfectionnées, forgent en partie le socle du troisième thème de ce numéro, Les moulages: un patrimoine, des techniques, des praticiens. L'histoire individuelle et professionnelle de ces artisans y est aussi privilégiée, avec la présentation des mouleurs Gherardi et Malzieux.

Enfin, les contributions rassemblées au sein de la dernière thématique, Les moulages, autres regards, autres pratiques, rendent compte du rôle des moulages dans le processus de création contemporaine. Elles illustrent également la diversité des usages et l'ampleur du champ géographique et chronologique couvert. Les moulages des basreliefs d'Abomey et de vestiges préhispaniques issus des collections du musée d'Ethnographie du Trocadéro participent de la réflexion sur les statuts et le sens dont ce type de collection est susceptible d'être investi.

7 Les contributions complémentaires au colloque, publiées ici, prennent toute leur place dans les quatre thèmes qui structurent ce volume. Elles en enrichissent les réflexions, rendent compte d'acquisitions récentes et actualisent la connaissance des collections ${ }^{1}$. 
8 Ce numéro est dédié à la mémoire de Marie-Paule Arnauld, conservateur général honoraire du patrimoine, qui porta avec force et dévouement la renaissance du musée des Monuments français et tout particulièrement celle de la galerie des moulages.

\section{NOTES}

1. - Nous souhaitons vivement remercier la rédaction de la revue et tout spécialement Françoise Cosler qui a suivi avec une bienveillante attention la création de ce numéro, ainsi que Catherine Gros dont la lecture attentive et la pertinence des remarques ont été saluées par tous les auteurs.

\section{AUTEURS}

\section{CHRISTINE LANCESTREMÈRE}

Conservatrice en chef du patrimoine, chef du service de la conservation, musée Rodin lancestremere@musee-rodin.fr

\section{JEAN-MARC HOFMAN}

Adjoint au conservateur de la Galerie des Moulages, Cité de l'Architecture et du Patrimoine, Musée des Monuments français jmhofman@citechaillot.fr

\section{YVES LE FUR}

Directeur du département du patrimoine et des collections, musée du quai Branly Yves.LEFUR@quaibranly.fr 